DAMTP-2005-78

hep-th/0509012

\title{
Kähler Moduli Inflation
}

\author{
Joseph P. Conlon ${ }^{1}$ and Fernando Quevedo ${ }^{2}$ \\ DAMTP, Centre for Mathematical Sciences, \\ Wilberforce Road, Cambridge, CB3 OWA, UK
}

\begin{abstract}
We show that under general conditions there is at least one natural inflationary direction for the Kähler moduli of type IIB flux compactifications. This requires a Calabi-Yau which has $h^{2,1}>h^{1,1}>2$ and for which the structure of the scalar potential is as in the recently found exponentially large volume compactifications. We also need - although these conditions may be relaxed - at least one Kähler modulus whose only nonvanishing triple-intersection is with itself and which appears by itself in the non-perturbative superpotential. Slow-roll inflation then occurs without a fine tuning of parameters, evading the $\eta$ problem of F-term inflation. In order to obtain COBE-normalised density perturbations, the stabilised volume of the Calabi-Yau must be $\mathcal{O}\left(10^{5}-10^{7}\right)$ in string units, and the inflationary scale $M_{\text {infl }} \sim 10^{13} \mathrm{GeV}$. We find a robust model independent prediction for the spectral index of $1-\frac{2}{N_{e}}=0.960 \rightarrow 0.967$, depending on the number of efoldings.
\end{abstract}

\footnotetext{
${ }^{1}$ e-mail: J.P.Conlon@damtp.cam.ac.uk

${ }^{2}$ e-mail: F.Quevedo@damtp.cam.ac.uk
} 


\section{Introduction}

One of the most exciting recent developments in string theory has been the great progress made in moduli stabilisation [1, 2]. There are now well-established techniques to give masses to the moduli that appear ubiquitously in string compactifications. The process of moduli stabilisation represents 'step zero' towards string phenomenology, as the moduli vevs determine such basic quantities as the string scale and the gauge coupling constants.

Given moduli potentials, an obvious application is to inflation. While the moduli sector has only indirect effects on Standard Model matter, the dynamics of light scalar fields is the principal theme of inflation. Inflation is the dominant paradigm for structure formation in the early universe and observations can now provide precision tests of inflationary models [3].

In string theory there are several candidates for the inflaton field, which can be classified according to their origin in either the open or closed string sector 4, 5. The most common open string inflaton is a brane/antibrane separation [6, 7, 8], whereas closed string inflatons typically correspond to geometric moduli 9]. There has been much recent effort devoted to inflationary model building, particularly since the appearance of the KKLT scenario of moduli stabilisation [2]. For recent discussions, 9, 10, 11, 12] may be consulted.

A standard problem bedevilling both brane and modular inflation - and indeed most supergravity inflation scenarios - is the $\eta$ problem. This states that for Fterm inflation the slow-roll $\eta$ parameter is $\mathcal{O}(1)$ unless a finely tuned cancellation occurs. The $\eta$ problem is manifest for F-term modular inflation. In brane inflation it is not manifest, but reappears once this is embedded into a moduli stabilisation scenario [10].

In this note we will present a simple inflationary scenario within the framework of the moduli stabilisation mechanism of [13, 14. The inflaton is one of the Kähler moduli and inflation proceeds by reducing the F-term energy. The $\eta$ problem is evaded by the pseudo-no scale property of the Kähler potential. The structure of the potential is such that inflation is obtained naturally and almost inevitably, without either fine tuning or a need to introduce large flux or brane numbers. This mechanism in principle applies to a very large class of Calabi-Yau compactifications that will be specified below.

\section{Almost Flat Directions}

\subsection{General Idea}

Slow-roll inflation requires the presence of almost flat directions in the scalar potential. A natural source of such a flat direction would be a field only appearing exponentially in the potential. Denoting this field by $\tau$, an appropriate (and 
textbook [15]) potential would be

$$
V_{i n f}=V_{0}\left(1-A e^{-\tau}+\ldots\right)
$$

where the dots represent higher exponents.

In string theory there are many moduli whose stabilisation requires nonperturbative effects. Examples are the Kähler moduli in IIB flux compactifications and both dilaton and Kähler moduli in heterotic Calabi-Yau compactifications. We regard all such fields as candidate inflatons, but shall focus on the Kähler moduli $\left(T_{i}\right)$ of type IIB flux compactifications. These only appear nonperturbatively in the superpotential, which takes the form

$$
W=\int G_{3} \wedge \Omega+\sum_{i} A_{i} e^{-a_{i} T_{i}},
$$

where $T_{i}=\tau_{i}+i c_{i}$ with $\tau_{i}$ the 4 -cycle volume and $c_{i}$ the axionic component. The $A_{i}$ represent threshold corrections and are independent of the Kähler moduli.

Of course, it is well known that $\mathcal{N}=1$ F-term inflation suffers from an $\eta$ problem. Both the Kähler potential and superpotential enter into the scalar potential, and for generic potentials $\eta \sim \mathcal{O}(1)$. However, the key word here is 'generic', and the Kähler potentials arising from string theory are (by definition) not generic. A common way these potentials fail to be generic is by being no-scale, corresponding to

$$
K^{i \bar{j}} \partial_{i} K \partial_{\bar{j}} K=3
$$

For a constant superpotential $W=W_{0}$, a no-scale scalar potential vanishes:

$$
V_{F}=e^{K}\left(K^{i \bar{j}} D_{i} W D_{\bar{j}} \bar{W}-3|W|^{2}\right)=0
$$

where $D_{i} W=\partial_{i} W+\left(\partial_{i} K\right) W$, with all directions being exactly flat. In type IIB, the tree-level Kähler potential for the size moduli takes the no-scale form

$$
\mathcal{K}=-2 \ln (\mathcal{V})
$$

where $\mathcal{V}$ is the internal volume. Suppose we now add nonperturbative modular dependence into the superpotential as in (2). The scalar potential becomes

$$
V_{F}=e^{K} K^{i \bar{j}}\left[a_{i} A_{i} a_{j} \bar{A}_{j} e^{-a_{i} T_{i}-a_{j} \bar{T}_{j}}-\left(\left(\partial_{i} K\right) W a_{j} \bar{A}_{j} e^{-a_{j} \bar{T}_{j}}+c . c\right)\right] .
$$

$T_{i}$ only appear nonperturbatively along exponentially flat directions and it is natural to ask whether this flatness can drive inflation.

While the potential (6) is exponentially flat, it also appears exponentially small. However, this is only true so long as all $T_{i}$ fields are large. In the presence of several Kähler moduli the variation of $V$ along the $T_{i}$ direction is in general 
uncorrelated with the magnitude of $V$ - we note this cannot happen in a onemodulus model.

There are also extra corrections to the potential, arising both from the breaking of no-scale behaviour by Kähler corrections and from the uplift terms needed to fine-tune the cosmological constant. The latter have several possible sources [2, 16, 17] and scale inversely with the volume

$$
V_{\text {uplift }} \sim \frac{1}{\mathcal{V}^{\alpha}}
$$

where $\frac{4}{3} \leq \alpha \leq 2$. Notice that the uplift encodes its modular dependence through the overall volume, rather than depending explicitly on the moduli. Thus at constant volume the $T_{n}$ direction is extremely flat for large values of $T_{n}$.

\subsection{Embedding in IIB Flux Compactifications}

While this is promising, inflation in string theory cannot be isolated from moduli stabilisation, as the methods used to stabilise the moduli can generate unacceptably large masses for the inflaton. We now embed the above in reasonably explicit IIB flux compactifications ${ }^{3}$ and in particular in the moduli stabilisation mechanism of [13, 14]. (For other recent work on perturbative corrections in IIB flux compactifications see [18, 19, 20, 21, 22]).

For multi-modulus Calabi-Yaus, evaluating the scalar potential requires expressing the overall volume in terms of the 4-cycle volumes, which we shall denote by $\tau_{i}=\operatorname{Re}\left(T_{i}\right)$. For illustration, we shall take a simplified form for the Calabi-Yau volume,

$$
\begin{aligned}
\mathcal{V} & =\alpha\left(\tau_{1}^{3 / 2}-\sum_{i=2}^{n} \lambda_{i} \tau_{i}^{3 / 2}\right) \\
& =\frac{\alpha}{2 \sqrt{2}}\left[\left(T_{1}+\bar{T}_{1}\right)^{3 / 2}-\sum_{i=1}^{n} \lambda_{i}\left(T_{i}+\bar{T}_{i}\right)^{3 / 2}\right] .
\end{aligned}
$$

$\tau_{1}$ controls the overall volume and $\tau_{2}, \ldots, \tau_{n}$ are blow-ups whose only non-vanishing triple intersections are with themselves. $\alpha$ and $\lambda_{i}$ are positive constants depending on the particular model. The minus signs are necessary as $\frac{\partial^{2} \mathcal{V}}{\partial T_{i} \partial T_{j}}$ must have signature $\left(1, h^{1,1}-1\right)[23$. We stabilise the dilaton and complex structure moduli

\footnotetext{
${ }^{3}$ The lack of explicitness lies principally in the difficulty of knowing whether and what nonperturbative superpotentials will be generated on a particular Calabi-Yau.
} 
with fluxes and take the Kähler moduli superpotential to be ${ }^{4}$

$$
W=W_{0}+\sum_{i=2}^{n} A_{i} e^{-a_{i} T_{i}}
$$

where $a_{i}=\frac{2 \pi}{g_{s} N}$. The Kähler potential is

$$
\mathcal{K}=\mathcal{K}_{c s}-2 \ln \left[\alpha\left(\tau_{1}^{3 / 2}-\sum_{i=2}^{n} \lambda_{i} \tau_{i}^{3 / 2}\right)+\frac{\xi}{2}\right],
$$

where $\xi=-\frac{\chi(M)}{2(2 \pi)^{3}}$. We have included the $\alpha^{\prime}$ corrections of [18]. The dilaton has been fixed and so we can define the moduli using either string or Einsteinframe volumes; we use the former. If the latter, we must replace $a_{i} \rightarrow a_{i} g_{s}$ and $\xi \rightarrow \xi g_{s}^{-3 / 2}$ in (9) and (10) - the physics is of course the same. As we work in the moduli stabilisaton framework of [13, 14] we anticipate that at the minimum we will have $\tau_{1}>>\tau_{i}$ and $\mathcal{V}>>1$. The resulting scalar potential is

$$
\left.V=e^{K}\left[G^{i \bar{j}} \partial_{i} W \partial_{\bar{j}} \bar{W}+G^{i \bar{j}}\left(\left(\partial_{i} K\right) W\right) \partial_{\bar{j}} \bar{W}+\text { c.c. }\right)\right]+\frac{3 \xi W_{0}^{2}}{4 \mathcal{V}^{3}} .
$$

We need $\xi>0$ and so require $h^{2,1}>h^{1,1}$. For the above Kähler potential, we have

$$
G^{i \bar{j}} \sim \frac{8 \mathcal{V} \sqrt{\tau_{i}}}{3 \alpha \lambda_{i}} \delta_{i j}+\mathcal{O}\left(\tau_{i} \tau_{j}\right)
$$

$G^{i \bar{j}}$ is real and, up to terms subleading in volume, satisfies $G^{i \bar{j}} \partial_{\bar{j}} \mathcal{K}=2 \tau_{i}$. At large volume only the leading part of $G^{i \bar{j}}$ is relevant and the scalar potential becomes

$$
V=\sum_{i} \frac{8\left(a_{i} A_{i}\right)^{2} \sqrt{\tau_{i}}}{3 \mathcal{V} \lambda_{i} \alpha} e^{-2 a_{i} \tau_{i}}-\sum_{i} 4 \frac{a_{i} A_{i}}{\mathcal{V}^{2}} W_{0} \tau_{i} e^{-a_{i} \tau_{i}}+\frac{3 \xi W_{0}^{2}}{4 \mathcal{V}^{3}}
$$

The minus sign in the second term arises from setting the $b_{i}$ axion to its minimum. There are terms not included in (13), but these are subleading. Importantly, they only depend on $\tau_{i}$ through the overall volume. This is crucial and ensures that at large $\tau_{i}$ the variation of the potential with $\tau_{i}$ is exponentially suppressed. We can find the global minimum by extremising (13) with respect to $\tau_{i}$. Doing this at fixed $\mathcal{V}$, we obtain

$$
\left(a_{i} A_{i}\right) e^{-a_{i} \tau_{i}}=\frac{3 \alpha \lambda_{i} W_{0}}{2 \mathcal{V}} \frac{\left(1-a_{i} \tau_{i}\right)}{\left(\frac{1}{2}-2 a_{i} \tau_{i}\right)} \sqrt{\tau_{i}} .
$$

${ }^{4}$ More generally we could take $W=W_{0}+\sum_{i=2}^{n} A_{i} e^{-a_{i j} T_{j}}$, which would alter the condition (18) in a model-dependent fashion. As long as the modified form of (18) can be satisfied, the results for the inflationary parameters are unaffected. In general we expect this to be possible, although we note that there do exist models, such as the $\mathcal{F}_{11}$ model of [24], for which this cannot be achieved. 
If we approximate $a_{i} \tau_{i}>>1$ (which is valid at large volume as $a_{i} \tau_{i} \sim \ln (\mathcal{V})$ ), then substituting this into the potential (13) contributes $\frac{-3 \lambda_{i} W_{0}^{2}}{2 \mathcal{V}^{3}} \tau_{i, \min }^{3 / 2} \alpha$, which can be reexpressed as $\frac{-3 \lambda_{i} W_{0}^{2} \alpha}{2 \mathcal{V}^{3} a_{i}^{3 / 2}}\left(\ln \mathcal{V}-c_{i}\right)^{3 / 2}$, where $c_{i}=\ln \left(\frac{3 \alpha \lambda_{i} W_{0}}{2 a_{i} A_{i}}\right)$. At large values of $\ln \mathcal{V}$, the resulting potential for the volume once all $\tau_{i}$ fields are minimised is

$$
V=\frac{-3 W_{0}^{2}}{2 \mathcal{V}^{3}}\left(\sum_{i=2}^{n}\left[\frac{\lambda_{i} \alpha}{a_{i}^{3 / 2}}\right](\ln \mathcal{V})^{3 / 2}-\frac{\xi}{2}\right) .
$$

This is the reason why the volume may be exponentially large. It is necessary to add an uplift term to ensure that the minimum is essentially Minkowksi. For concreteness we use IASD fluxes ${ }^{5}$ and write the volume potential as

$$
V=\frac{-3 W_{0}^{2}}{2 \mathcal{V}^{3}}\left(\sum_{i=2}^{n}\left[\frac{\lambda_{i} \alpha}{a_{i}^{3 / 2}}\right](\ln \mathcal{V})^{3 / 2}-\frac{\xi}{2}\right)+\frac{\gamma W_{0}^{2}}{\mathcal{V}^{2}}
$$

where $\gamma \sim \mathcal{O}\left(\frac{1}{\mathcal{V}}\right)$ parametrises the magnitude of the uplift. By tuning $\gamma$, the potential (16) (and by extension its full form (13)) has a Minkowski or small de Sitter minimum.

To obtain inflation we consider the potential away from the minimum. We take a 'small' modulus, say $\tau_{n}$, as the inflaton and displace it far from its minimum. At constant volume the potential is exponentially flat along this direction, and the modulus rolls back in an inflationary fashion. There is no problem in terms of initial conditions. While we do not know how the moduli evolution starts, we do know how it must end, namely with all moduli at their minima. Given this - we have nothing new to say on the overshoot problem [25] - inflation occurs as the last Kähler modulus rolls down to its minimum.

It is necessary that all other moduli, and in particular the volume, are stable during inflation. Displacing $\tau_{n}$ from its minimum nullifies the contribution made by the stabilised $\tau_{n}$ to the volume potential. The effective volume potential during inflation is then

$$
V=\frac{-3 W_{0}^{2}}{2 \mathcal{V}^{3}}\left(\sum_{i=2}^{n-1}\left[\frac{\lambda_{i} \alpha}{a_{i}^{3 / 2}}\right](\ln \mathcal{V})^{3 / 2}-\frac{\xi}{2}\right)+\frac{\gamma W_{0}^{2}}{\mathcal{V}^{2}}
$$

Provided that the ratio

$$
\rho \equiv \frac{\lambda_{n}}{a_{n}^{3 / 2}}: \sum_{i=2}^{n} \frac{\lambda_{i}}{a_{i}^{3 / 2}}
$$

is sufficiently small ${ }^{6}$, there is little difference between (16) and (17) and the

\footnotetext{
${ }^{5}$ These are pure supergravity and so it is manifest that the uplift only depends on the volume with unwanted dependence on $\tau_{i}$.

${ }^{6}$ This can be quantified in explicit models. For large volumes the condition on the ratio $\rho$ is that $9.5(\ln \mathcal{V}) \rho<1$. As long as we restrict to reasonable values for the $a_{i}$, this bounds the volume at the minimum. To obtain inflation with correct density perturbations, the appropriate volumes are $\mathcal{O}\left(10^{5}-10^{7}\right)$, which can be satisfied using sensible values for $\lambda_{i}$ and $a_{i}$.
} 
volume modulus will be stable during inflation. As we obviously require $\rho<1$, it follows that at least three Kähler moduli are necessary. While (18) can always be satisfied by an appropriate choice of $a_{i}$, this becomes easier and easier with more Kähler moduli.

We illustrate the form of the resulting inflationary potential in figure 1, showing the inflaton and volume directions.

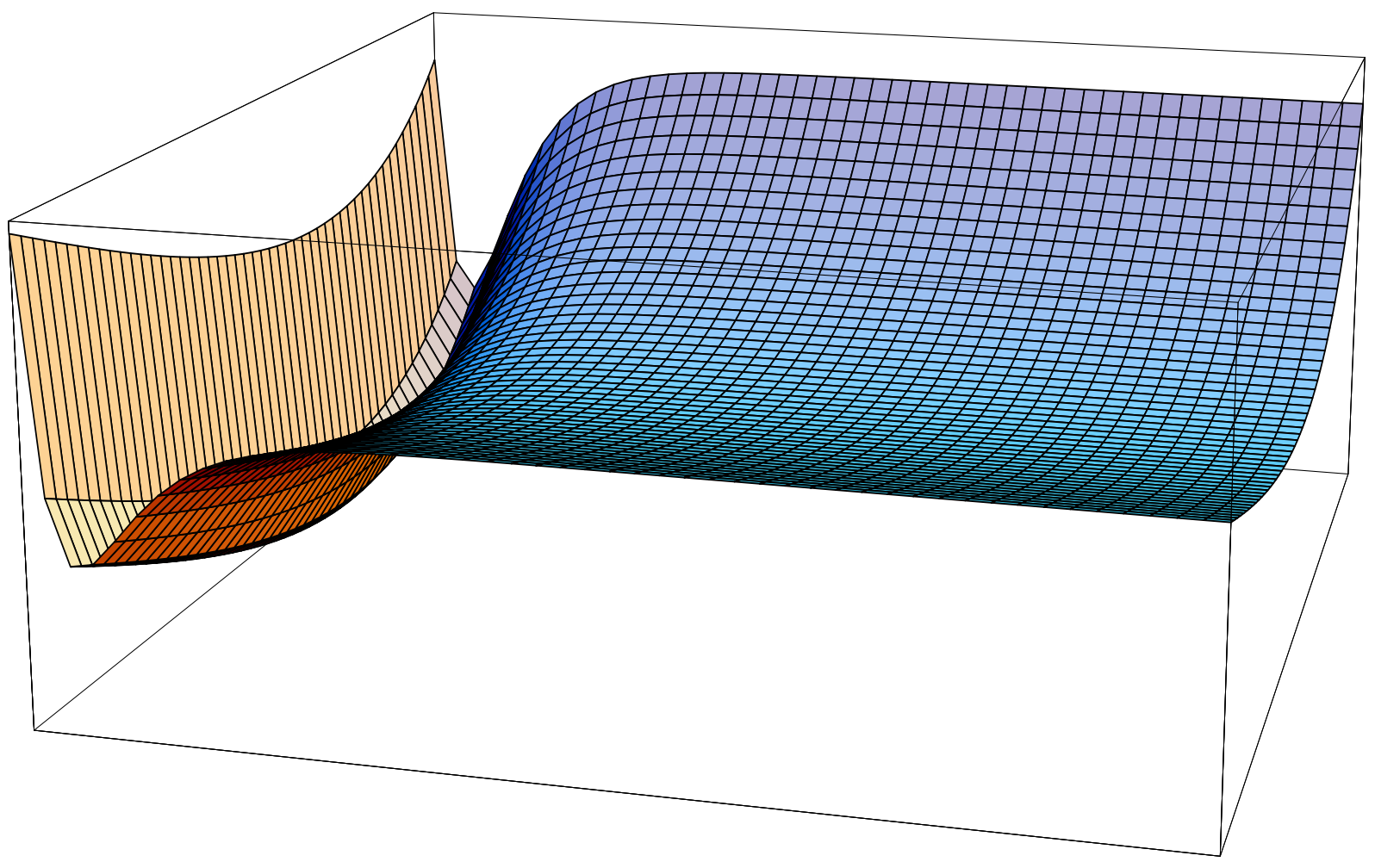

Figure 1: Inflationary potential: the inflaton lies along the x-direction and the volume along the y-direction.

\section{Inflationary Potential and Parameters}

Let us now quantify the resulting potential and compute the inflationary parameters. The inflationary potential is read off from (13) to be

$$
V_{\text {inf }}=V_{0}-\frac{4 \tau_{n} W_{0} a_{n} A_{n} e^{-a_{n} \tau_{n}}}{\mathcal{V}^{2}}
$$

as the double exponential in (13) is irrelevant during inflation. During inflation $V_{0}$ is constant and can be parametrised as

$$
V_{0}=\frac{\beta W_{0}^{2}}{\mathcal{V}^{3}}
$$


$\left(\frac{1}{\mathcal{V}^{3}}\right.$ is the scale of the potential during inflation). However, $\tau_{n}$ is not canonically normalised, as to leading order in volume

$$
K_{n \bar{n}}=\frac{3 \lambda}{8 \sqrt{\tau_{n}}}
$$

The canonically normalised field is

$$
\tau_{n}^{c}=\sqrt{\frac{4 \lambda}{3 \mathcal{V}}} \tau_{n}^{\frac{3}{4}}
$$

In terms of $\tau_{n}^{c}$, the inflationary potential is

$$
V=V_{0}-\frac{4 W_{0} a_{n} A_{n}}{\mathcal{V}^{2}}\left(\frac{3 \mathcal{V}}{4 \lambda}\right)^{2 / 3}\left(\tau_{n}^{c}\right)^{4 / 3} \exp \left[-a_{n}\left(\frac{3 \mathcal{V}}{4 \lambda}\right)^{2 / 3}\left(\tau_{n}^{c}\right)^{4 / 3}\right]
$$

This is similar, but not identical, to the textbook potential $V=V_{0}\left(1-e^{-\tau}\right)$. Although $\tau_{n}^{c}$ is canonically normalised, it has no natural geometric interpretation and for clarity we shall express the inflationary parameters in terms of $\tau_{n}$, the cycle volume.

The slow-roll parameters are defined by

$$
\begin{aligned}
\epsilon & =\frac{M_{P}^{2}}{2}\left(\frac{V^{\prime}}{V}\right)^{2}, \\
\eta & =M_{P}^{2} \frac{V^{\prime \prime}}{V} \\
\xi & =M_{P}^{4} \frac{V^{\prime} V^{\prime \prime \prime}}{V^{2}}
\end{aligned}
$$

with the derivatives being with respect to $\tau_{n}^{c}$. These can be evaluated to give

$$
\begin{aligned}
\epsilon & =\frac{32 \mathcal{V}^{3}}{3 \beta^{2} W_{0}^{2}} a_{n}^{2} A_{n}^{2} \sqrt{\tau_{n}}\left(1-a_{n} \tau_{n}\right)^{2} e^{-2 a_{n} \tau_{n}} \\
\eta & =-\frac{4 a_{n} A_{n} \mathcal{V}^{2}}{3 \lambda \sqrt{\tau_{n}} \beta W_{0}}\left[\left(1-9 a_{n} \tau_{n}+4\left(a_{n} \tau_{n}\right)^{2}\right) e^{-a_{n} \tau_{n}}\right] \\
\xi & =\frac{-32\left(a_{n} A_{n}\right)^{2} \mathcal{V}^{4}}{9 \beta^{2} \lambda^{2} W_{0}^{2} \tau_{n}}\left(1-a_{n} \tau_{n}\right)\left(1+11 a_{n} \tau_{n}-30\left(a_{n} \tau_{n}\right)^{2}+8\left(a_{n} \tau_{n}\right)^{2}\right) e^{-2 a_{n} \tau_{n}}
\end{aligned}
$$

Then $\xi<<\epsilon, \eta<<1$ provided that $e^{-a_{n} \tau_{n}}<<\frac{1}{\mathcal{V}^{2}}$.

Within the slow-roll approximation, the spectral index and its running are given by

$$
\begin{aligned}
n-1 & =2 \eta-6 \epsilon+\mathcal{O}(\xi) \\
\frac{d n}{d \ln k} & =16 \epsilon \eta-24 \epsilon^{2}-2 \xi
\end{aligned}
$$


The number of efoldings is given by

$$
N_{e}=\int_{\phi_{\text {end }}}^{\phi} \frac{V}{V^{\prime}} d \phi
$$

which may be expressed as

$$
N_{e}=\frac{-3 \beta W_{0} \lambda_{n}}{16 \mathcal{V}^{2} a_{n} A_{n}} \int_{\tau_{n}^{\text {end }}}^{\tau_{n}} \frac{e^{a_{n} \tau_{n}}}{\sqrt{\tau_{n}}\left(1-a_{n} \tau_{n}\right)} d \tau_{n} .
$$

Matching the COBE normalisation for the density fluctuations $\delta_{H}=1.92 \times 10^{-5}$ requires

$$
\frac{V^{3 / 2}}{M_{P}^{3} V^{\prime}}=5.2 \times 10^{-4}
$$

where the LHS is evaluated at horizon exit, $N_{e}=50-60$ efoldings before the end of inflation. This condition can be expressed as

$$
\left(\frac{g_{s}^{4}}{8 \pi}\right) \frac{3 \lambda \beta^{3} W_{0}^{2}}{64 \sqrt{\tau_{n}}\left(1-a_{n} \tau_{n}\right)^{2}}\left(\frac{W_{0}}{a_{n} A_{n}}\right)^{2} \frac{e^{2 a_{n} \tau_{n}}}{\mathcal{V}^{6}}=2.7 \times 10^{-7}
$$

We have here included a factor of $\frac{g_{s}^{4}}{8 \pi}$ that should properly be included as an overall normalisation in $V$ - see [14. The condition (32) determines the normalisation of the potential and in practice we use it as a constraint on the stabilised volume.

Finally, the tensor-to-scalar ratio is

$$
r \sim 12.4 \epsilon
$$

\subsection{Footprint of the Model}

We now want to determine the inflationary predictions for the above model. In the above model there are various undetermined parameters arising from the detailed microphysics, such as the threshold correction $A$ or tree-level superpotential $W_{0}$. In principle, these are determined by the specific Calabi-Yau with its brane and flux configurations, but they can be prohibitively difficult to calculate in realistic examples. However, it turns out that the most important results are independent of these parameters. In particular, solving equations (28) to (34) numerically, we find the robust results

$$
\begin{aligned}
\eta & \approx-\frac{1}{N_{e}} \\
\epsilon & <10^{-12} \\
\xi & \approx-\frac{2}{N_{e}^{2}}
\end{aligned}
$$


These results are not so surprising given the similarity of the potential to the textbook form $V_{0}\left(1-e^{-\tau}\right)$. Taking a range of $N_{e}=50 \rightarrow 60$, we obtain in the slow-roll approximation

$$
\begin{aligned}
0.960 & <n<0.967 \\
-0.0006 & <\frac{d n}{d \ln k}<-0.0008 \\
0 & <|r|<10^{-10}
\end{aligned}
$$

where the uncertainties above arise principally from the number of e-foldings. If we go beyond the slow-roll approximation, the expression for $n$ will receive $\mathcal{O}(\xi)$ corrections - these are minimal and can be neglected.

To evaluate the inflationary energy scale, it is convenient to reformulate the COBE normalisation of density perturbations $\delta_{H}=1.92 \times 10^{-5}$ as

$$
\frac{V^{1 / 4}}{\epsilon^{1 / 4}}=6.6 \times 10^{16} \mathrm{GeV} \text {. }
$$

Unlike the predictions for the spectral index, the required internal volume is parameter-dependent. For typical values of the microscopic parameters this is found numerically to take a range of values

$$
10^{5} l_{s}^{6} \leq \mathcal{V} \leq 10^{7} l_{s}^{6}
$$

where $l_{s}=(2 \pi) \sqrt{\alpha^{\prime}}$. As the moduli stabilisation mechanism of [13, 14] naturally generates exponentially large volumes, there is no difficulty in achieving these values. The range of $\epsilon$ at horizon exit is $10^{-13} \geq \epsilon \geq 10^{-15}$, and thus the inflationary energy scale is rather low,

$$
V_{\text {inf }} \sim 10^{13} \mathrm{GeV} \text {. }
$$

This implies in particular that tensor perturbations would be unobservable in this model.

There is no practical upper limit on the number of efoldings attainable. This is large-field inflation and the potential is exponentially flat as the inflaton 4-cycle increases in volume. A very large number of efoldings is achieved by a very small variation in the inflaton and barring cancellations we would expect $N_{e, t o t a l}>>60$ in these models.

In these compactifications, the lightest non-axionic modulus has a mass [14]

$$
M \sim \frac{M_{P}}{\mathcal{V}^{3 / 2}}
$$

Thus even at the larger end of volumes $M>>\mathcal{O}(10) \mathrm{TeV}$ and there is no cosmological moduli problem. As indicated earlier, there is also not a problem with initial conditions for inflation. Given that the moduli attain their minimum, the 
inflaton is simply the last Kähler modulus to roll down to the minimum. We do not need to worry about interference from the evolution of the other moduli. Once they roll down to the minimum they become heavy and will rapidly decouple from inflationary dynamics.

We have nothing new to say on the cosmological overshoot problem. It is difficult to see how progress may be achieved here without an adequate formulation of initial conditions for the universe. This problem is amplified by the fact that typical Calabi-Yaus have hundreds of both complex structure and Kähler moduli; it is very difficult to give a well-motivated choice for the initial values and evolution of so many moduli. (For the possibility that damping can remove the overshoot problem see [26, 27, 28,).

\subsection{Additional Corrections and Extensions}

The inflationary mechanism presented here relies on the exponential flatness of the $\tau_{n}$ direction at constant volume. This is unbroken by the tree-level Kähler potential, the $\left(\alpha^{\prime}\right)^{3}$ correction and the uplift term. Let us briefly discuss effects that might spoil this.

Let us first focus on superpotential effects. The nonrenormalisation theorems guarantee that the Kähler moduli cannot appear perturbatively in $W$. However, the flatness could be spoiled if the gauge kinetic functions $A_{i}$ depended polynomially on the Kähler moduli. A term $A\left(T_{j}\right) e^{-T_{i}}$ in the superpotential would lead to an effective polynomial term for $T_{j}$ once $T_{i}$ was stabilised. However, the $A_{i}$ must be holomorphic in $T_{i}$ and respect the axion shift symmetries, and so this polynomial dependence on $T_{i}$ cannot occur. Indeed, in models for which the threshold corrections have been computed explicitly, there is no dependence of the gauge kinetic functions on the Kähler moduli [19]. Combined with nonrenormalisation results, this means that the exponential flatness cannot be lifted by superpotential effects.

The other possibility is that the exponential flatness may be lifted by corrections to the Kähler potential that depend on $\tau_{n}$. Considering first bulk terms, both the tree-level Kähler potential and the $\mathcal{O}\left(\alpha^{\prime 3}\right)$ correction computed in [18] have the property that their contribution to the scalar potential is only a function of the volume and has no explicit dependence on the moduli. These then do not affect the constant volume flatness of the $\tau_{n}$ direction. It would be interesting, but difficult, to determine whether this feature extends beyond the terms so far computed.

There are also open string Kähler corrections such as those recently computed in [19]. Of necessity, this computation is restricted to certain toroidal orientifolds with D3 and D7 branes. The 1-loop corrections determined there are subdominant in the scalar potential to the $\mathcal{O}\left(\alpha^{\prime 3}\right)$ corrections, although they give a larger contribution to the Kähler potential. This counterintuitive result is due to the 
fact that a Kähler correction

$$
K+\delta K=-2 \ln (\mathcal{V})+\frac{\epsilon}{\mathcal{V}^{2 / 3}}
$$

only gives in the scalar potential

$$
\frac{\delta V}{V}=\frac{\mathcal{O}(\epsilon)}{\mathcal{V}^{4 / 3}}
$$

Thus $\mathcal{O}\left(\mathcal{V}^{-2 / 3}\right)$ corrections to the Kähler potential are in fact subdominant in the scalar potential to $\mathcal{O}\left(\mathcal{V}^{-1}\right)$ corrections. This result makes the volume stabilisation mechanism of [13, 14] more robust. For the models for which the string loop computation can be performed, there is no analogue of the $\tau_{n}$ blow-up field and so it is unclear whether and in what fashion these might appear in the 1-loop correction to the Kähler potential. There are however physical constraints: $e^{K}$ appears in the scalar potential and so must behave sensibly in the limits of both small and large $\tau_{n}$.

There are also field theory loop corrections determined in 20]. These are again subdominant in the scalar potential to the $\mathcal{O}\left(\alpha^{\prime 3}\right)$ corrections used above for volume stabilisation. This computation again does not have an analogue of the blow-up modes we have used for the inflaton.

The upshot is that the exponential flatness of the $\tau_{n}$ direction is not broken by any of the known corrections. In general, any correction that can be expressed in terms of the overall volume will not alter the exponential flatness of the $\tau_{n}$ direction. If corrections existed which did break this exponential flatness, it would be necessary to examine their form and magnitude - it is not after all necessary that the exponential flatness survive for all values of $\tau_{n}$, but merely for those relevant during the last sixty e-folds.

Finally, we have used an oversimplified form for the Calabi-Yau, picturing it as simply a combination of a volume cycle and blow-up modes. This is not necessary for the inflationary mechanism described here. Whilst in (8) we assumed $h^{1,1}-1$ moduli to be blow-ups whose only nonvanishing triple intersection was with themselves, a single such modulus would be perfectly adequate as an inflaton. Indeed, even this is not necessary - the minimal requirement is simply a flat direction, which originates from the no-scale behaviour and is broken by nonperturbative effects. The condition necessary to ensure the volume is stable during inflaton will then be a generalisation of (18).

\section{Discussion}

We have presented a general but simple scenario of inflation in string theory that does not require fine tuning of parameters, applies to a very large class of compactifications and is predictive at the level that can be ruled out within a few years. 
This scenario realises large field inflation in a natural way. The main properties of these models are the existence of flat directions broken by non-perturbative effects. The flat directions have their origins in the no-scale property of the Kähler potential and are generic for IIB Kähler moduli, as is the appearance of instanton-generated nonperturbative superpotentials. The scenario is embedded in the exponentially large volume compactifications of [13, 14, and requires $h^{2,1}>h^{1,1}$ and $h^{1,1}>2$. This last requirement is necessary to ensure that the volume is stabilised during inflation.

Notice that the volumes required to obtain inflation, while large, are not extremely large as the string scale is only a few orders of magnitude below the Planck scale. The necessary volumes of $\mathcal{O}\left(10^{5}-10^{7}\right)$ in string units can be obtained by natural choices of the exponential parameters $a_{i}\left(a \sim \frac{2 \pi}{3}\right.$ in the simplest cases) [14.

Although there are many moduli, the inflationary period reduces to a singlefield case. This is because the inflaton is simply the last modulus to roll down to its minimum, and once other moduli attain their minimum they rapidly become heavy and decouple from inflationary dynamics. In principle there are at least two other fields that may have a nontrivial role during the cosmological evolution. One is the axion partner of the inflaton field. We have chosen this to sit at the minimum of its oscillatory potential, at least for the last sixty efolds. This is not a strong assumption - because the inflaton direction is so flat, there is a lot of time for the axion to relax from a possibly non-zero inital value to its minimum before the last sixty efolds start. (Remember that depending on initial conditions the total number of efolds may be many orders of magnitude larger than 60.) It would nonetheless be interesting to study a multiple field inflation configuration in which both fields contribute to the density perturbations [29].

There is also a second direction which is extremely flat, corresponding to the axionic partner of the overall volume modulus. This field is so light, with $m<<10^{-300} \mathrm{GeV}[14$ both during and after inflation, that it will not play a role in the cosmological evolution.

We have considered inflation as occurring at the top of a waterfall, and inflation ending as the moduli roll down to the waterfall. It may also be interesting to consider the case where there are multiple waterfalls. By appropriately tuning the uplift we could arrange that the current vacuum energy corresponds to the top of a waterfall rather than the bottom. As the field would be slowly rolling this would then correspond to quintessence.

Another open question concerns reheating. Note that unlike brane inflation, in which reheating is driven by tachyon condensation [6] requiring non-trivial string theory dynamics to be understood [30, in our case, as in racetrack inflation, reheating is a pure field theory problem that only requires the study of the matter/inflaton couplings. In this respect, if the standard model lies on D7 branes wrapping the four-cycle whose size is determined by the inflaton field, the inflaton can decay directly to the gauge fields of the standard model through the 
coupling $\tau_{n} F^{\mu \nu} F_{\mu \nu}$. This can give rise to efficient reheating as discussed in e.g. 31. If the standard model lies elsewhere, the inflaton will couple to standard model fields through higher dimension operators and a more detailed analysis is required. (For a recent analysis of reheating in brane-antibrane inflation see [32]).

It is worth comparing aspects of this mechanism with other inflationary models obtained from string theory. Besides the issue of fine tuning, it differs from racetrack inflation and tachyon driven inflation in that it corresponds to large field rather than hill-top inflation. In principle our scenario is closer to brane separation inflation, but we do not need a second field to end inflation and in particular do not predict the existence of remnant cosmic strings from the reheating era. Numerically, our predictions are close to racetrack inflation although with the spectral index within a more comfortable range. The exponentially flat direction resembles the mechanism of [8], with the advantage that moduli stabilisation is now derived and not assumed.

Let us finally discuss the generality of our scenario. The main technical assumption we have used is the direct expression for the volume in terms of the Kähler moduli (8) . This was overkill - the only part of the assumption we actually used was that the inflaton modulus appears alone in the volume as $\mathcal{V}=\ldots-\left(T_{n}+\bar{T}_{n}\right)^{\frac{3}{2}}$. As indicated above, we can relax even this: the absolute minimal requirement is simply the existence of a flat direction broken by nonperturbative effects. There may be several possible inflationary directions in the above model, $\tau_{2}, \ldots, \tau_{n}$ are all good candidates - with the particular one chosen determined by which Kähler modulus is last to attain its minimum. In each case we expect similar physics to emerge with a robust prediction on the spectral index of density perturbations. It is very exciting that such a simple string scenario has the basic properties needed for a realisation of cosmological inflation with predictions that can be confirmed or ruled out in the near future.

\section{Acknowledgements}

We acknowledge useful conversations with P. Berglund, C. Burgess, S. Kachru and B. van Tent. The research of FQ is partially funded by PPARC and a Royal Society Wolfson merit award. JC is grateful to EPSRC for a research studentship.

\section{References}

[1] S. B. Giddings, S. Kachru and J. Polchinski, "Hierarchies from fluxes in string compactifications," Phys. Rev. D 66, 106006 (2002) arXiv:hep-th/0105097. 
[2] S. Kachru, R. Kallosh, A. Linde and S. P. Trivedi, "De Sitter vacua in string theory," Phys. Rev. D 68, 046005 (2003) arXiv:hep-th/0301240.

[3] C. L. Bennett et al., "First Year Wilkinson Microwave Anisotropy Probe (WMAP) Observations: Preliminary Maps and Basic Results," Astrophys. J. Suppl. 148 (2003) 1 arXiv:astro-ph/0302207.

[4] P. Binetruy and M. K. Gaillard, "Candidates For The Inflaton Field In Superstring Models," Phys. Rev. D 34 (1986) 3069; T. Banks, M. Berkooz, S. H. Shenker, G. W. Moore and P. J. Steinhardt, "Modular cosmology," Phys. Rev. D 52 (1995) 3548 arXiv:hep-th/9503114.

[5] G. R. Dvali and S. H. H. Tye, "Brane inflation," Phys. Lett. B 450, 72 (1999) arXiv:hep-ph/9812483.

[6] C. P. Burgess, M. Majumdar, D. Nolte, F. Quevedo, G. Rajesh and R. J. Zhang, "The inflationary brane-antibrane universe," JHEP 0107, 047 (2001) arXiv:hep-th/0105204.

[7] G. R. Dvali, Q. Shafi and S. Solganik, "D-brane inflation," arXiv:hep-th/0105203.

[8] C. P. Burgess, P. Martineau, F. Quevedo, G. Rajesh and R. J. Zhang, "Brane antibrane inflation in orbifold and orientifold models," JHEP 0203, 052 (2002) arXiv:hep-th/0111025.

[9] J. J. Blanco-Pillado et al., "Racetrack inflation," JHEP 0411, 063 (2004) arXiv:hep-th/0406230.

[10] S. Kachru, R. Kallosh, A. Linde, J. Maldacena, L. McAllister and S. P. Trivedi, "Towards inflation in string theory," JCAP 0310 (2003) 013 arXiv:hep-th/0308055.

[11] J. P. Hsu, R. Kallosh and S. Prokushkin, "On brane inflation with volume stabilization," JCAP 0312 (2003) 009 arXiv:hep-th/0311077; F. Koyama, Y. Tachikawa and T. Watari, "Supergravity analysis of hybrid inflation model from D3-D7 system", arXiv:hep-th/0311191; H. Firouzjahi and S. H. H. Tye, "Closer towards inflation in string theory," Phys. Lett. B 584 (2004) 147 arXiv:hep-th/0312020. J. P. Hsu and R. Kallosh, "Volume stabilization and the origin of the inflaton shift symmetry in string theory," JHEP 0404 (2004) 042 arXiv:hep-th/0402047;

[12] C. P. Burgess, J. M. Cline, H. Stoica and F. Quevedo, "Inflation in realistic D-brane models," arXiv:hep-th/0403119; J. M. Cline and H. Stoica, "Multibrane inflation and dynamical flattening of the inflaton potential," arXiv:hep-th/0508029. O. DeWolfe, S. Kachru and H. Verlinde, 
"The giant inflaton," JHEP 0405 (2004) 017 arXiv:hep-th/0403123. N. Iizuka and S. P. Trivedi, "An inflationary model in string theory," arXiv:hep-th/0403203. K. Becker, M. Becker and A. Krause, "M-theory inflation from multi M5-brane dynamics," Nucl. Phys. B 715 (2005) 349 arXiv:hep-th/0501130 . D. Cremades, F. Quevedo and A. Sinha, "Warped tachyonic inflation in type IIB flux compactifications and the open-string completeness conjecture," arXiv:hep-th/0505252. S. Dimopoulos, S. Kachru, J. McGreevy and J. G. Wacker, "N-flation," arXiv:hep-th/0507205. E. Silverstein and D. Tong, "Scalar speed limits and cosmology: Acceleration from D-cceleration," Phys. Rev. D 70 (2004) 103505 arXiv:hep-th/0310221]; M. Alishahiha, E. Silverstein and D. Tong, "DBI in the sky," Phys. Rev. D 70 (2004) 123505 arXiv:hep-th/0404084|; X. g. Chen, "Inflation from warped space," arXiv:hep-th/0501184 H. Singh, "(A)symmetric tachyon rolling in de Sitter spacetime: A universe devoid of Planck density," arXiv:hep-th/0508101.

[13] V. Balasubramanian, P. Berglund, J. P. Conlon and F. Quevedo, "Systematics of moduli stabilisation in Calabi-Yau flux compactifications," JHEP 0503 (2005) 007 arXiv:hep-th/0502058.

[14] J. P. Conlon, F. Quevedo and K. Suruliz, "Large-volume flux compactifications: Moduli spectrum and D3/D7 soft supersymmetry breaking," arXiv:hep-th/0505076.

[15] A. R. Liddle and D. H. Lyth, "Cosmological inflation and large-scale structure," CUP (2000).

[16] C. P. Burgess, R. Kallosh and F. Quevedo, "de Sitter string vacua from supersymmetric D-terms," JHEP 0310, 056 (2003) arXiv:hep-th/0309187|.

[17] A. Saltman and E. Silverstein, JHEP 0411, 066 (2004) arXiv:hep-th/0402135.

[18] K. Becker, M. Becker, M. Haack and J. Louis, "Supersymmetry breaking and alpha'-corrections to flux induced potentials," JHEP 0206, 060 (2002) arXiv:hep-th/0204254.

[19] M. Berg, M. Haack and B. Kors, "Loop corrections to volume moduli and inflation in string theory," Phys. Rev. D 71, 026005 (2005) arXiv:hep-th/0404087. . M. Berg, M. Haack and B. Kors, "String Loop Corrections to Kahler Potentials in Orientifolds," arXiv:hep-th/0508043. M. Berg, M. Haack and B. Kors, "On Volume Stabilization by Quantum Corrections," arXiv:hep-th/0508171.

[20] G. von Gersdorff and A. Hebecker, "Kaehler corrections for the volume modulus of flux compactifications," arXiv:hep-th/0507131. 
[21] V. Balasubramanian and P. Berglund, "Stringy corrections to Kaehler potentials, SUSY breaking, and the cosmological constant problem," JHEP 0411, 085 (2004) arXiv:hep-th/0408054. P. Berglund and P. Mayr, "Non-perturbative superpotentials in F-theory and string duality," arXiv:hep-th/0504058.

[22] K. Bobkov, "Volume stabilization via alpha' corrections in type IIB theory with fluxes," JHEP 0505, 010 (2005) arXiv:hep-th/0412239|. A. Westphal, "Eternal inflation with alpha' corrections," arXiv:hep-th/0507079.

[23] P. Candelas and X. de la Ossa, "Moduli Space Of Calabi-Yau Manifolds," Nucl. Phys. B 355, 455 (1991).

[24] F. Denef, M. R. Douglas and B. Florea, "Building a better racetrack," JHEP 0406, 034 (2004) arXiv:hep-th/0404257.

[25] R. Brustein and P. J. Steinhardt, "Challenges for superstring cosmology," Phys. Lett. B 302, 196 (1993) arXiv:hep-th/9212049.

[26] N. Kaloper and K. A. Olive, "Dilatons in string cosmology," Astropart. Phys. 1 (1993) 185.

[27] N. Kaloper, J. Rahmfeld and L. Sorbo, "Moduli entrapment with primordial black holes," Phys. Lett. B 606, 234 (2005) arXiv:hep-th/0409226|.

[28] R. Brustein, S. P. de Alwis and P. Martens, "Cosmological stabilization of moduli with steep potentials," Phys. Rev. D 70 (2004) 126012 arXiv:hep-th/0408160.

[29] B. van Tent, "Multiple-field inflation and the CMB," Class. Quant. Grav. 21 (2004) 349 arXiv:astro-ph/0307048.

[30] G. Shiu, S. H. H. Tye and I. Wasserman, "Rolling tachyon in brane world cosmology from superstring field theory," Phys. Rev. D 67 (2003) 083517 arXiv:hep-th/0207119; J. M. Cline, H. Firouzjahi and P. Martineau, "Reheating from tachyon condensation," JHEP 0211 (2002) 041 arXiv:hep-th/0207156|; N. Barnaby, C. P. Burgess and J. M. Cline, "Warped reheating in brane-antibrane inflation," JCAP 0504 (2005) 007 arXiv:hep-th/0412040|; L. Kofman and P. Yi, "Reheating the universe after string theory inflation," arXiv:hep-th/0507257, A. R. Frey, A. Mazumdar and R. Myers, "Stringy Effects During Inflation and Reheating," arXiv:hep-th/0508139.

[31] A. D. Linde, "Particle Physics and Inflationary Cosmology," Harwood (1990) 362 p. (Contemporary concepts in physics, 5) arXiv:hep-th/0503203. 
[32] D. Chialva, G. Shiu and B. Underwood, "Warped reheating in multi-throat brane inflation," arXiv:hep-th/0508229. 